\title{
Evaluation of a Culturally Adapted Training in Indigenous Mental Health and Wellbeing for the Alcohol and Other Drug Workforce
}

\author{
Rachael Hinton and Tricia Nagel \\ Wellbeing and Chronic Disease Division, Menzies School of Health Research, P.O. Box 41096, Casuarina, NT 0811, Australia \\ Correspondence should be addressed to Tricia Nagel, tricia.nagel@menzies.edu.au
}

Received 2 December 2011; Accepted 3 January 2012

Academic Editors: P. Bendtsen, E. Braehler, W. B. Hansen, and B. Vicente

Copyright (C) 2012 R. Hinton and T. Nagel. This is an open access article distributed under the Creative Commons Attribution License, which permits unrestricted use, distribution, and reproduction in any medium, provided the original work is properly cited.

Indigenous Australians have high rates of mental illness comorbid with substance misuse. The complex needs of this client group create challenges for the alcohol and other drug (AOD) workforce. This paper describes the outcomes of an Indigenous-specific "Yarning about Mental Health" training for the AOD workforce to strengthen knowledge and skills in mental health approaches and in their engagement with Indigenous clients. The training provides culturally adapted strategies and tools for understanding mental health, promoting wellbeing, and delivering brief interventions in the substance misuse setting. A nonexperimental evaluation which incorporated pre-post questionnaires was conducted with workshop participants attending one of four trainings. The training was perceived to be highly appropriate and helpful in participants' work with Indigenous AOD clients. There was significant improvement in confidence and knowledge related to Indigenous mental health and wellbeing and qualitative data supported these positive outcomes. This study supports the need to blend Indigenous concepts of health and wellbeing with nonIndigenous ways of understanding and treating illness in order to develop services which are appropriate to Indigenous peoples. It also suggests research is required to understand whether self-reported increases in knowledge and confidence can translate into behavioural changes in participants' teaching and practice of culturally competent care and to improved client outcomes.

\section{Introduction}

Mental illness is associated with substantial disability, yet many people with mental disorders do not seek help from any health professional for their mental health problems [1]. In Australia, Aboriginal and Torres Strait Islander people are even less likely than non-Indigenous people to access treatment [2], with a contributing factor being that some existing services lack the capacity to take account of Indigenous conceptualisations of health and wellbeing [3]. In using the term Indigenous we refer to Aboriginal and Torres Strait Islander people and acknowledge their diversity of language and culture. Indigenous people do not necessarily distinguish between wellbeing and "physical" health or "mental" health or "cultural" or "spiritual" wellbeing; instead, they have a "whole of life" view [4]. Furthermore, Indigenous Australians have high rates of mental illness comorbid with substance misuse. It is particularly important, therefore, to provide mental health care at first point of contact, especially in alcohol and other drug (AOD) treatment settings.

Alcohol and other drug services are reporting a steady increase in the number of Indigenous clients with comorbid mental health problems [5], many of whom are at higher risk of relapse, self-harm, and suicide [6]. The complex needs of this client group create challenges for the AOD workforce in terms of engagement, resources and skills [7]. The need to build the capacity of this heterogeneous workforce (which includes non-AOD health professionals, social professionals; AOD specialist health professionals, such as psychologists and counselors; AOD workers and nonhealth professionals, e.g., managers) in mental health care is well documented $[5,8-11]$, and resources and training have proliferated in the past decade aligned with changing policy priorities [12]. 
Australian guidelines on the management of cooccurring alcohol and other drug and mental health conditions provide evidence-based information to assist with the management of comorbid AOD and mental health conditions [11, 13]. Standardised tools such as Psycheck can be used by the AOD workforce to screen for symptoms of mental health conditions in their clients [14] and an Indigenous-specific assessment, the Indigenous risk impact Screen (IRIS), assists with detection of AOD problems and some mental health risks [15]. Despite these resources, it remains a challenge for AOD services to empower consumers [16] and provide recovery oriented client-centred care that respects lived experience, promotes mutual self-help, is holistic and looks beyond symptoms and illness [17]. A specific challenge for these services is the need to adapt treatment approaches to different cultural contexts. Many Indigenous people live in remote and rural areas with limited access to specialist mental health services [18] and marked differences exist in literacy, language, and worldview [19] which further complicates accurate and sensitive assessment and treatment. In the light of these difficulties, there is a need to develop culturally adapted psychological interventions that can be delivered in AOD settings.

Studies among New Zealand Māori AOD treatment workers have shown that both Māori and Western knowledge bases and practices are seen as not only equally relevant but also complementary in the treatment of addiction for Maori people. Accordingly, it is recommended that specific training for this workforce integrate Māori and Western knowledge, skills and processes of learning [20]. Similarly in Australia, Indigenous-specific mental health training is needed for the AOD workforce which integrates Indigenous cultural frameworks and recognises the historical, cultural, and political forces that affect the health of Indigenous people [21]. The priority is the delivery of culturally appropriate health care, which acknowledges and promotes cultural identity, links with traditional ways of communication and incorporates different understandings of health and identity [22].

The Aboriginal and Islander Mental health initiative (AIMhi) has developed a brief, culturally adapted intervention for strengthening mental health and wellbeing in Indigenous communities [23]. The AIMhi approach promotes consumer empowerment. It applies a recovery focus through therapeutic goal setting, motivation, and recognition of the family and community context of the individual client. AIMhi developed tools and resources that support each step of engagement of Indigenous people in services: mental health literacy, assessment, screening, early intervention, treatment, and outcome measurement. The Indigenous-specific training program, "Yarning about Mental Health", integrates the tools developed in the AIMhi program and is provided by a five person training team, three of whom are Indigenous. The name of the training incorporates a commonly used word in parts of Australia for having an informal conversation, that is, "to have a yarn." The training is delivered in flexible workshop formats (two hour, half-day, full-day, and two-day training) in either urban centres or remote communities. It incorporates different learning styles (uses simple English, audio, pictorial, and interactive methods), literacy levels, and Indigenous cultural frameworks, including a holistic concept of health and wellbeing. It was developed to provide service provider training in cross-cultural approaches to mental health with the aim of translating research outcomes into specialist and primary care settings. The Yarning about Mental Health training has since been adapted to different health settings including chronic disease and alcohol and other drug treatment. As a result, demand for training and resources have increased across a broad range of practitioners including youth services, perinatal, and child and maternal health services.

The authors recognise that education and training comprise only a part of workforce development, taking equal places with support strategies for skills and knowledge and workplace structure and policy [9]. This paper focuses on describing the outcomes of Yarning about Mental Health training, the first stage (2008-2010) of a workforce development project which provided Indigenous-specific training for the AOD workforce to strengthen knowledge and skills in recovery approaches and in their engagement with Indigenous clients. The second stage of the project (2008 to 2010) provided a train the trainer component for participants of the Yarning about Mental Health training, while the third and final stage, completed in 2011, examined the application of the training in practice. The aim of the current study was to measure the effectiveness of the one day Yarning about Mental Health training in strengthening the knowledge and confidence of the AOD workforce in mental health assessment, screening, early intervention, and treatment specific to their Indigenous clients.

\section{Methods}

2.1. Description of the Training. The Yarning about Mental Health training is embedded within a consumer empowerment and recovery approach and provides culturally appropriate strategies and tools for understanding mental health, promoting wellbeing and delivering brief interventions in the setting of mental illness, substance misuse, and physical illness. The content is described in the "Yarning About Mental Health" training manual [24] and covers the following: Indigenous concepts of mental health and wellbeing, strengths-based care planning, early warning signs, goal setting, risk assessment, mental state examination, and medication. Participants are trained in the use of strengths based approaches and resources and materials are designed to be used specifically with Indigenous people, including the "mental health stay strong care plan package," flip charts and information sheets on depression, mania, psychosis, dementia, delirium, and medication [23].

From 2008 to 2010, four Yarning about Mental Health one-day training workshops were conducted with members of Western Australian Network of Alcohol and other Drug Agencies (WANADA) and Network of Alcohol and Drug Agencies (NADA). Each workshop was followed by a oneday train the trainer workshop. The training was in response to a request from both networks to address the identified 
gap in knowledge, confidence, and skills within the AOD workforce in addressing mental health and wellbeing issues specifically among Indigenous clients. Indigenous and nonIndigenous participants attended one of the four workshops held in Kununurra and Albany in Western Australia and in Sydney, New South Wales.

2.2. Ethics. The project received approval from the Human Research Ethics Committee of the Northern Territory Department of Health and Families and the Menzies School of Health Research. Informed consent was obtained from participants prior to the pre-workshop evaluation. Confidentiality was maintained by the allocation of a unique identification number, necessary for matching pre- and postworkshop responses.

2.3. Evaluation. The design was a nonexperimental evaluation which incorporated pre-post questionnaires with workshop participants attending the Yarning about Mental Health training workshops. A structured questionnaire incorporating ordinal scales and open-ended questions complemented the quantitative data to provide further insight into participant's perspectives and experiences. Participants completed questionnaires at the beginning of the training (pre-workshop) and at the end of the training (postworkshop). The findings of post-training questionnaires administered immediately following the train the trainer workshop as well as 12-24 months after the workshop are outside the scope of the paper and a detailed discussion of this data is forthcoming.

The pre-workshop questionnaire included sociodemographic questions and examined experience, training, and current client load. Participants were asked about confidence in assessment and treatment of Indigenous people with mental illness and their knowledge of causes, treatment, and early warning signs of mental health problems, specifically for Indigenous people. Knowledge and confidence were rated on a scale from 1 (not much/not confident) to 9 (a lot/very confident). Participants were also asked to rate their use of AIMhi resources on a scale from 1 (not at all) to 5 (all of the time). The post-workshop questionnaire included the same questions with additional questions rating the training on a scale of 1 (not at all) to 4 (very) in terms of usefulness, interest, and building of cross-cultural partnerships.

2.4. Analysis. Analysis was conducted using the statistical data analysis program "STATA" [25] and Wilcoxon's signed rank tests were used to compare trainees' mental health knowledge and confidence at baseline and after training. Summative scales rated knowledge and confidence on a scale of 1 to 9 as low (1-3), medium (4-6), and high (7-9).

\section{Results}

3.1. Characteristics of Participants. Fifty-nine members of the AOD workforce participated in four workshops and the majority (75\%) of participants was female. Most participants identified as AOD workers or AOD counsellors, mental
TABle 1: Participant self-reported workplace experience and training.

\begin{tabular}{|c|c|}
\hline Participant characteristics & $\begin{array}{c}\text { Value } n \\
(\%)\end{array}$ \\
\hline Female* & $43(75.4)$ \\
\hline \multicolumn{2}{|l|}{ Service provider role } \\
\hline AOD worker/AOD counsellor & $12(20.3)$ \\
\hline Mental health worker & $10(16.9)$ \\
\hline Allied health worker & $10(16.9)$ \\
\hline Coordinator/manager & $7(11.8)$ \\
\hline Case worker/counsellor & $7(11.8)$ \\
\hline Aboriginal mental health worker & $4(6.7)$ \\
\hline Other & $8(15.2)$ \\
\hline \multicolumn{2}{|l|}{ Number of current clients ${ }^{\#}$} \\
\hline $0-5$ & $24(41.4)$ \\
\hline $6-10$ & $15(25.9)$ \\
\hline $11-20$ & $8(13.8)$ \\
\hline$>20$ & $9(15.5)$ \\
\hline Unknown & $1(1.7)$ \\
\hline \multicolumn{2}{|l|}{ Formal training in mental health } \\
\hline$<2$ weeks & $14(23.7)$ \\
\hline 2 weeks to 3 months & $12(20.3)$ \\
\hline$>3$ months & $32(54.2)$ \\
\hline Unknown & $1(1.7)$ \\
\hline \multicolumn{2}{|l|}{$\begin{array}{l}\text { Years experience working in } \\
\text { Indigenous mental health }^{\dagger}\end{array}$} \\
\hline$<2$ years & $30(51.7)$ \\
\hline $2-5$ years & $11(19)$ \\
\hline$>5$ years & $17(29.3)$ \\
\hline \multicolumn{2}{|l|}{$\begin{array}{l}\text { Work in partnership with } \\
\text { Indigenous colleagues }{ }^{\ddagger}\end{array}$} \\
\hline None & $5(8.9)$ \\
\hline Little & $11(19.6)$ \\
\hline Some & $7(12.5)$ \\
\hline Most & $9(16.1)$ \\
\hline All the time & $24(42.9)$ \\
\hline
\end{tabular}

health and allied health workers. Although over half of participants had formal mental health training and worked in partnership with Indigenous colleagues, most were relatively new to the care of Indigenous clients (Table 1).

3.2. Knowledge and Confidence. Fifty-nine participants completed the pre- and post-workshop questionnaire. Pre- to post-workshop comparison of confidence and knowledge showed significant change in all measures excluding "knowledge of the cause of mental illness" (Table 2). Post-workshop participants reported improved knowledge of the warning signs and treatment of mental illness and in levels of confidence to assess, treat and communicate with Indigenous mental health clients. There was a significant increase in 
TABLe 2: Participant mental health knowledge and confidence ${ }^{\mathrm{a}}$.

\begin{tabular}{lccc}
\hline Subscale & $\begin{array}{c}\text { Pre-training } \\
M(\mathrm{SD})\end{array}$ & $\begin{array}{c}\text { Post-training } \\
M(\mathrm{SD})\end{array}$ & Wilcoxon $z(P)$ \\
\hline $\begin{array}{l}\text { Know about causes } \\
\text { Know about }\end{array}$ & $5.77(1.78)$ & $7.28(1.43)$ & $-1.199(0.2306)$ \\
$\begin{array}{l}\text { treatment } \\
\text { Know early }\end{array}$ & $5.87(2.11)$ & $6.91(1.74)$ & $-2.931(0.034)$ \\
$\begin{array}{l}\text { warning signs } \\
\text { Confidence to } \\
\text { communicate }\end{array}$ & $5.78(2.06)$ & $6.98(1.65)$ & $-3.251(0.0012)$ \\
$\begin{array}{l}\text { Confidence to } \\
\text { assess }\end{array}$ & $4.96(1.97)$ & $6.81(1.65)$ & $-4.511(0.0000)$ \\
Confidence to treat $4.79(2.10)$ & $6.64(1.88)$ & $-4.453(0.0000)$ \\
\hline
\end{tabular}

${ }^{a}$ Scale 1-9. Higher score indicative of a greater level of confidence and knowledge of mental health.

confidence in treatment and assessment irrespective of participants' level of training and experience.

Participants $(n=15)$ described increased access to practical tools and resources for use with Indigenous clients, as an Allied Health Worker explained:

[Itwas a] really good engaging workshop... developed really usable skills \& engendered confidence in working with Aboriginal and Torres Strait Islander people with mental health concerns.

The training helped participants to overcome lack of confidence in working cross-culturally with comorbid clients as an AOD counsellor highlighted:

I have learnt a lot from this training and feel very confident now to work with people.... It is not fear based anymore.

3.3. Usefulness and Interest in AIMhi Resources. At baseline, the majority of participants $(n=36,63 \%)$ were either not aware of or not regularly using any AIMhi resources. AIMhi flipcharts $(n=19,33 \%)$ were the resources most commonly used, followed by AIMhi information sheets $(n=$ $16,28 \%)$. All of the participants agreed after the workshop that the training would be beneficial and useful in their work with Indigenous clients (Table 3). In particular 81\% of participants found the workshop very interesting and $88 \%$ indicated that the resources were very helpful. Many $(60 \%)$ of the participants commented that the culturally appropriate strategies and resources would promote better communication and understanding between the AOD workforce and Indigenous clients; for example:

[I was] very impressed with the resources-our client group will understand and feel included in the process (AOD counsellor).

The majority of participants (89\%) reported that the training and resources would contribute to building confidence and knowledge in the assessment and treatment of
TABLe 3: Participant post-workshop ratings of the Yarning about Mental Health training.

\begin{tabular}{|c|c|c|c|}
\hline Item & $\begin{array}{c}\text { A little } \\
n(\%)\end{array}$ & $\begin{array}{l}\text { Some } \\
n(\%)\end{array}$ & $\begin{array}{l}\text { A lot } \\
n(\%)\end{array}$ \\
\hline Workshop was interesting & & $11(19)$ & $46(81)$ \\
\hline Resources are useful & & $7(12)$ & $50(88)$ \\
\hline $\begin{array}{l}\text { Training and resources build } \\
\text { my confidence and knowledge }\end{array}$ & & $6(11)$ & $51(89)$ \\
\hline $\begin{array}{l}\text { Training will help me to build } \\
\text { cross cultural partnerships }\end{array}$ & $1(2)$ & $10(17)$ & $46(81)$ \\
\hline
\end{tabular}

mental illness among Indigenous AOD clients. Participants were also asked to consider the extent to which the training and resources would help to build cross-cultural partnerships in assessment and treatment of mental illness. The majority of participants (81\%) responded that the training and resources would be beneficial, for example,

The more information the better and trying this different educational material, it would benefit myself, clients and community (Aboriginal mental health worker).

Several participants $(n=7)$ noted that the training and resources would also contribute to the creation of a more mutually beneficial and less intimidating relationship between client and practitioner:

This is something I can use easily... I have had trouble getting clients to come up with smaller realistic goals and small steps they can make rather than do everything and when I suggest things to them they feel frustrated (Aboriginal Mental Health Worker).

3.4. Experience of the Workshop and Knowledge Translation. The final two questions of the post-workshop questionnaire asked participants to consider their experience of the workshop and opportunities for change in practice. Fiftyone $(86 \%)$ participants responded to the question on their experience and the majority $(n=49)$ gave a response that aggregated to one of two main themes: satisfaction with the process of the workshop and usefulness and applicability of training content. Positive comments from participants about the process related to the workshop structure, the interactive and engaging delivery, the benefits of having a mix of Indigenous and non-Indigenous participants and the apparent experience and knowledge of the trainers, including the inclusion of Aboriginal co-trainers:

Fantastic. Interesting informative, well presented. Tools are colourful well thought out \& a delight to use. The knowledge skills and experience of the facilitators shines through (Role unknown).

Excellent quality training and training style. Great to have Aboriginal co-trainers. Loved the 


\section{simplicity of how the resource is delivered and used} (AOD worker).

Participants' responses to workshop applicability related to the culturally-specific information, tools, strategies, and resources that were introduced and examined as part of the training. This practical approach appeared to help to demystify and simplify the management of mental health and wellbeing concerns of Indigenous AOD clients, and in turn, provide participants with new skills, knowledge and confidence to apply their learning in practice. As several participants explained:

I really enjoyed the training. I have been thinking on easy ways to engage with teenagers and I believe this will really be useful. It was clear, simple, to the point and it provided me with tools that increase my confidence with the therapy (Mental Health Worker).

Five participants suggested ways to improve the training, which in all cases related to practical outcomes and the application of the tools. Their responses could also reflect the different experience and knowledge among the professional mix of participants and the challenge of tailoring the training to meet this diversity:

It was valuable, informative and helpful, but a little slow. There could be far more focus on the tool and its use. The tool is simple and streamlined, the training could be too (Mental Health Worker).

Three main themes emerged from participants' responses ( $n=47$ ) to opportunities for practice change: the confirmation of their intended use of the tools $(n=26,55 \%)$; integration of the tools with existing resources and strategies ( $n=9,19 \%)$ to increase their options for the management of individual clients and enhance current practice; and continuing to build a better understanding and relationships ( $n=16,34 \%$ ) between AOD workers and Indigenous clients with AOD and mental health comorbidities.

\section{Discussion}

Culturally appropriate intervention strategies in Indigenous mental health can contribute to countering the disproportionate burden of disease in the Indigenous population through increasing accessing to services [13]. This study finds that culturally adapted brief mental health training was perceived to be highly appropriate and helpful in participants' work with Indigenous AOD clients. The results show that the AOD workforce was initially low in confidence in assessment and treatment of Indigenous peoples with mental illness. Pre- to post-workshop scores highlight significant improvement in confidence and knowledge related to Indigenous mental health and qualitative data supported these positive outcomes. These findings are similar to other studies that have shown culturally adapted training, education, and resources can improve provider knowledge, self, efficacy [26], and competency [27].
In the current context of high prevalence of emotional distress among Indigenous AOD clients, there is a particular need to resource and train the AOD workforce. This includes a need to blend Indigenous concepts of health and wellbeing and non-Indigenous ways of understanding and treating illness in order to develop services which are appropriate to Indigenous peoples [28-30] and to integrate recovery oriented approaches to mental health care. The overall findings of this study indicate that this culturally-specific client-centred training strengthened participants' knowledge and skills in collaborative mental health care. The training also met the needs, interests, and perspectives of a diverse group of participants and was seen to be appropriate for AOD workers who have different experiences and levels of training.

Despite a number of important findings, the results should be interpreted in the context of the study's limitations. The majority of participants reported that they would change their practice as a result of the training, although the findings do not show the extent to which the training is sustained and implemented as part of routine clinical practice or whether it contributed to improved client outcomes. Mentoring, observation, and supervision of participants post-training was outside the scope of this project and other studies have shown that training of AOD practitioners in screening for mental health disorders, combined with supervision, improved detection rates, and the quality of interventions in AOD services [14]. The sample was also small and restricted to a specific sector, limiting the generalisability of the findings.

\section{Conclusion}

The study provides support for the use of AIMhi resources and approach to Indigenous mental health within the AOD workforce. The training is useful and applicable for experienced practitioners as well as those new to mental health. In the field of AOD workforce development, an important question for future research is whether selfreported increases in knowledge and confidence translate to changes in participants' teaching and practice of culturally competent care and improved client outcomes.

\section{Acknowledgments}

The authors would like to acknowledge the contribution of Mr. Neil Spencer to this paper and thank the AIMhi trainers and the participants of the four Yarning about Mental Health trainings.

\section{References}

[1] T. Slade, A. Johnston, M. A. Oakley Browne, G. Andrews, and H. Whiteford, "2007 National Survey of Mental Health and Wellbeing: methods and key findings," Australian and New Zealand Journal of Psychiatry, vol. 43, no. 7, pp. 594-605, 2009.

[2] H. Whiteford and A. Groves, "Policy implications of the 2007 Australian National Survey of Mental Health and Wellbeing," 
Australian and New Zealand Journal of Psychiatry, vol. 43, no. 7, pp. 644-651, 2009.

[3] T. Westerman, "Guest editorial: engagement of Indigenous clients in mental health services," Australian e-Journal for the Advancement of Mental Health, vol. 3, no. 3, 2004.

[4] National Aboriginal and Torres Strait Islander Health Council, National Strategic Framework for Aboriginal and Torres Strait Islander Health-Framework for Action by Governments, Canberra, Australia, 2003.

[5] D. Gray, M. Green, S. Saggers et al., Review of the Aboriginal and Torres Strait Islander Community-Controlled Alcohol and other Drugs Sector in Queensland, National Drug Research Institute, Perth, Australia, 2009.

[6] A. Roche and Y. Pollard, Improved Services for People with Drug and Alcohol Problems and Mental Illness: Assisting Alcohol and other Drugs (AOD) Non-Government Organisations to Better Respond to People with Co-Morbid AOD and Mental Health Issues, National Centre for Education and Training on Addiction (NCETA), Flinders University, Adelaide, Australia, 2006.

[7] S Berry and T. Crowe, "A review of engagement of Indigenous Australians within mental health and substance abuse services," Australian e-Journal for the Advancement of Mental Health, vol. 8, no. 1, 2009.

[8] K. Wolinski, M. O'Neill, A. Roche et al., Workforce Issues and the Treatment of Alcohol Problems: A Survey of Managers of Alcohol and Drug Treatment Agencies, Australian Government Department of Health and Ageing, Canberra, Australia, 2003.

[9] A. Roche and J. McDonald, Systems, Settings, People: Workforce Development Challenges for the Alcohol and Other Drugs Field, National Centre for Education and Training on Addiction, Flinders University, Adelaide, Australia, 2003.

[10] L. Hides, D. I. Lubman, K. Elkins, L. S. Catania, and N. Rogers, "Feasibility and acceptability of a mental health screening tool and training programme in the youth alcohol and other drug (AOD) sector," Drug and Alcohol Review, vol. 26, no. 5, pp. 509-515, 2007.

[11] K. Mills, M. Deady, H. Proudfoot et al., Guidelines on the Management of Co-Occurring Alcohol and other Drug and Mental Health Conditions in Alcohol and other Drug Treatment Settings, National Drug and Alcohol Research Centre, University of New South Wales, Sydney, Australia, 2009.

[12] L. Pearce and J. Savage, "Workforce issues for indigenous substance use," in Systems, Settings, People: Workforce Development Challenges for the Alcohol and Other Drugs Field, A. Roche and J. McDonald, Eds., National Centre for Education and Training on Addiction, Adelaide, Australia, 2003.

[13] DoHA, Alcohol Treatment Guidelines for Indigenous Australians, Canberrra, Australia, 2007.

[14] N. Lee, L. Jenner, A. Baker et al., "Screening and intervention for mental health problems in alcohol and other drug settings: can training change practitioner behaviour?" Drugs, vol. 18, no. 2, pp. 157-160, 2011.

[15] C. M. Schlesinger, C. Ober, M. M. McCarthy, J. D. Watson, and A. Seinen, "The development and validation of the Indigenous Risk Impact Screen (IRIS): a 13-item screening instrument for alcohol and drug and mental health risk," Drug and Alcohol Review, vol. 26, no. 2, pp. 109-117, 2007.

[16] D. Rickwood, Pathways of Recovery: Preventing Further Episodes of Mental Illness (Monograph), Canberra, Australia, 2006.

[17] S. Onken, J. Dumont, and P. Ridgway, Mental Health Recovery: What Helps and What Hinders? A National Research Project for the Development of Recovery Facilitating System Performance Indicators, National Technical Assistance Center for State
Mental Health Planning and the National Association of State Mental Health Program Directors, Alexandria, Va, USA, 2002.

[18] R. Bailey, F. Siciliano, G. Dane, and al. et, Atlas of Health Related Infrastructure in Discrete Indigenous Communities, ATSIC, Melbourne, Australia, 2002.

[19] T. Westerman, "Engagement of Indigenous clients in mental health services: what role do cultural differences play?" Australian e-Journal for the Advancement of Mental Health, vol. 3, no. 3, 2004.

[20] P. Robertson, T. Gibson, and S. Adamson, "Initial results for the 2004/05 national telephone survey of Māori alcohol and other drug (AOD) treatment workers," in New Zealand Addiction Treatment Research Monograph, S. Adamson, Ed., Proceedings from the Cutting Edge Conference, 2005.

[21] L. M. Hart, A. F. Jorm, L. G. Kanowski, C. M. Kelly, and R. L. Langlands, "Mental health first aid for Indigenous Australians: using Delphi consensus studies to develop guidelines for culturally appropriate responses to mental health problems," BMC Psychiatry, vol. 9, article 47, 2009.

[22] T. Nagel and C. Thompson, "AIMHI NT "Mental Health Story Teller Mob": developing stories in mental health," Australian e-Journal for the Advancement of Mental Health, vol. 6, no. 2, 2007.

[23] T. Nagel, C. Thompson, N. Spencer et al., "Two way approaches to Indigenous mental health training: brief training in brief interventions," Australian e-Journal for the Advancement of Mental Health, vol. 8, no. 2, 2009.

[24] T. Nagel and C. Thompson, Yarning About Mental Health Manual, Australian Integrated Mental Health Initiative, Darwin, Australia, 2006.

[25] Statacorp, Stata Statistical Software, Stata Corporation, College Station, Tex, USA, 9th edition, 2006.

[26] M. Y. Yu, L. Song, A. Seetoo, C. Cai, G. Smith, and D. Oakley, "Culturally competent training program: a key to training lay health advisors for promoting breast cancer screening," Health Education and Behavior, vol. 34, no. 6, pp. 928-941, 2007.

[27] D. Wilson, "The significance of a culturally appropriate health service for Indigenous Ma-ori women," Contemporary Nurse, vol. 28, no. 1-2, pp. 173-188, 2008.

[28] L. Kirmayer, C. Simpson, and M. Cargo, "Healing traditions: culture, community and mental health promotion with Canadian Aboriginal peoples," Australasian Psychiatry, vol. 11, supplement 1, pp. S15-S23, 2003.

[29] V. McLennan and F. Khavarpour, "Culturally appropriate health promotion: its meaning and application in Aboriginal communities," Health Promotion Journal of Australia, vol. 15, no. 3, pp. 237-239, 2004.

[30] R. Murray, K. Bell, J. Elston et al., Guidelines for Development, Implementation and Evaluation of National Public Health Strategies in Relation to ATSI Peoples, National Public Health Partnership, Melbourne, Australia, 2002. 


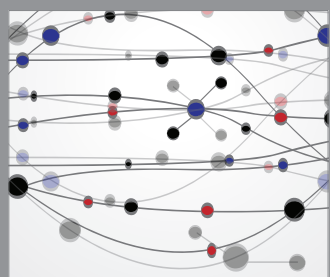

The Scientific World Journal
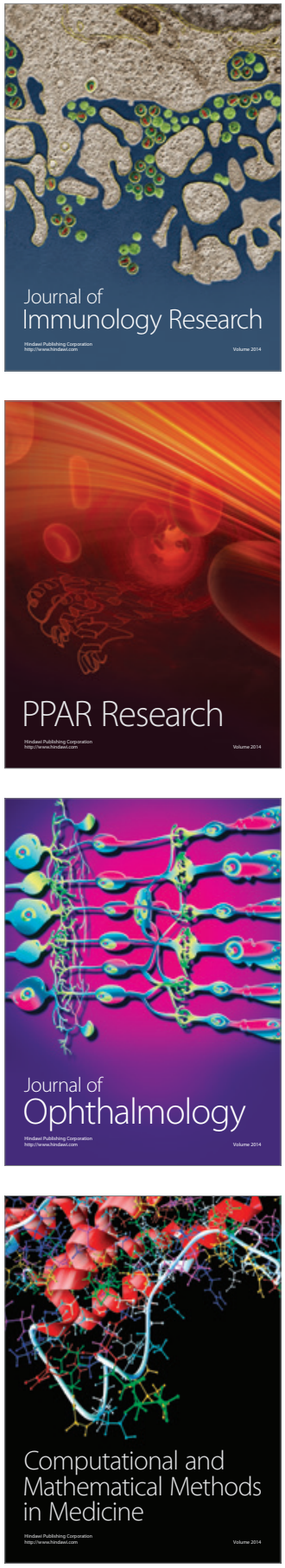

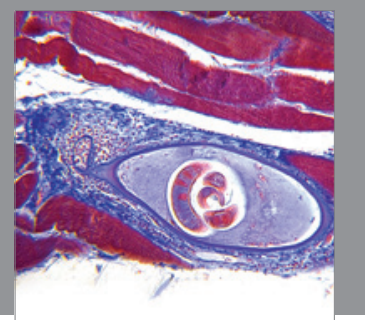

Gastroenterology

Research and Practice
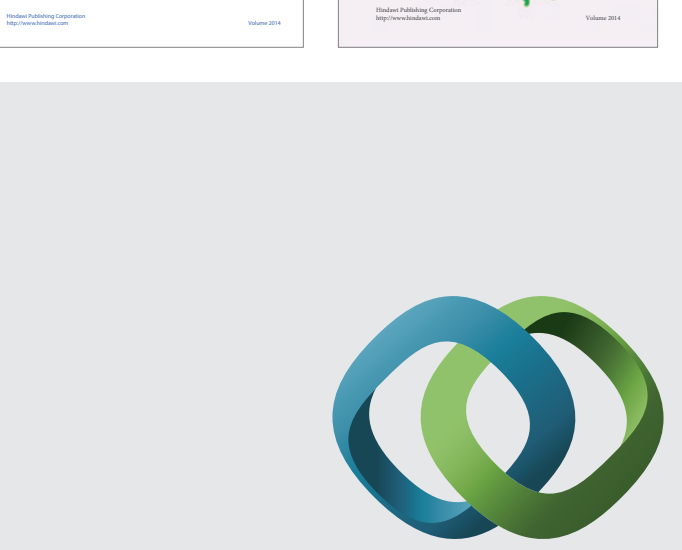

\section{Hindawi}

Submit your manuscripts at

http://www.hindawi.com
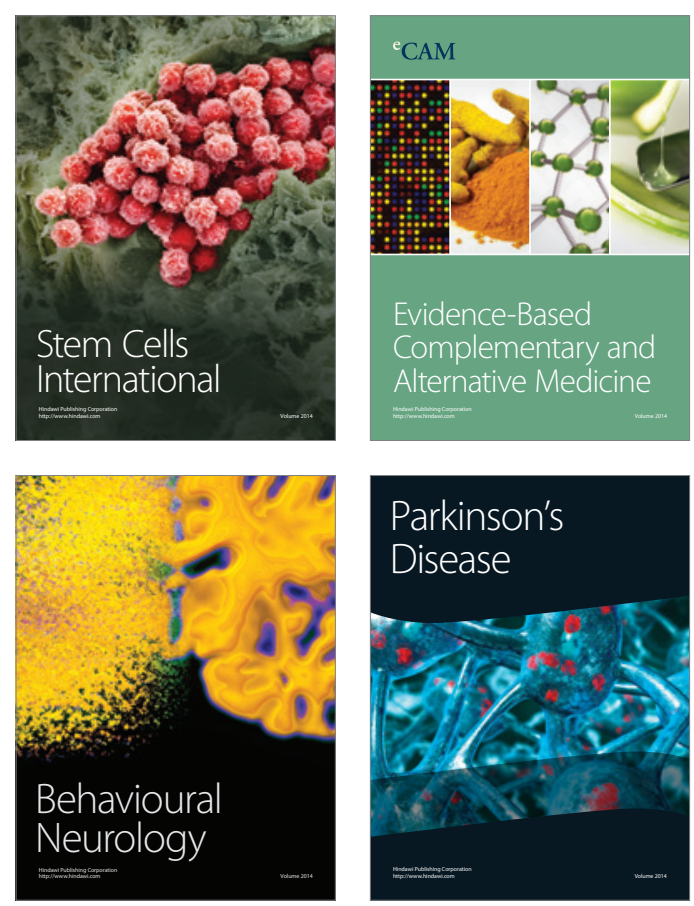

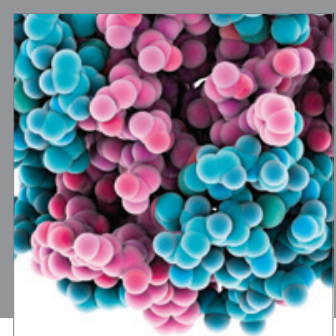

Journal of
Diabetes Research

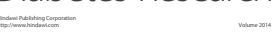

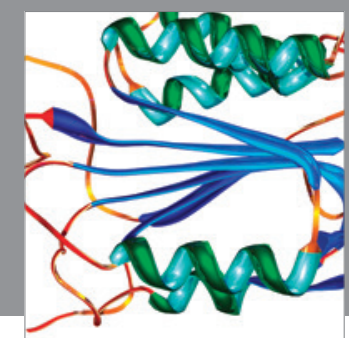

Disease Markers
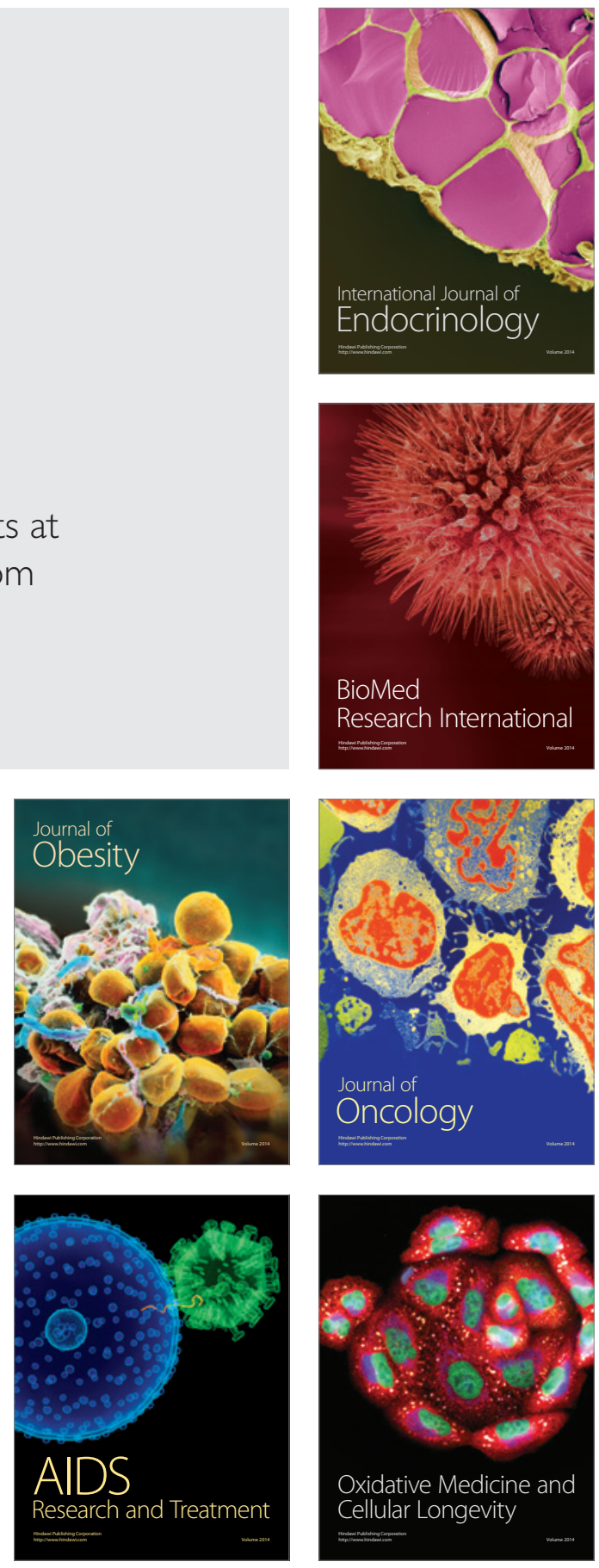